\title{
From Pure Geomatics for Algebraic Procedures with a View to Obtaining Equations from Ellipse from the Perspective of René Descartes
}

\author{
Ezequias Adolfo Domingas Cassela ${ }^{*}$, Amado Leonardo André \\ Department of Exact Sciences, Bié Higher Pedagogical School, Cuito/Bié, Angola \\ Email address: \\ ezequiasadolfo@hotmail.com (E. A. D. Cassela),amadoleonardo2009@hotmail.com (A. L. André) \\ ${ }^{*}$ Corresponding author
}

\section{To cite this article:}

Ezequias Adolfo Domingas Cassela, Amado Leonardo André. From Pure Geomatics for Algebraic Procedures with a View to Obtaining Equations from Ellipse from the Perspective of René Descartes. Pure and Applied Mathematics Journal. Vol. 10, No. 4, 2021, pp. 89-95. doi: $10.11648 /$ j.pamj.20211004.11

Received: July 19, 2021; Accepted: July 28, 2021; Published: August 4, 2021

\begin{abstract}
This article aims to study the ellipse from the perspective of pure or synthetic geometry to the representation of points on a plane through the use of real numbers, as well as the representation and classification of this conic curve through the use of equations. The perspective developed in this article is based on the view of René Descartes, in considering that "the algebraic steps in a demonstration should really correspond to a geometric representation." The relevance of this article is to bring a reflection that eliminates the study of Analytical Geometry through ready-made and finished formulas, without satisfactory justification and without a logical chain that gives a greater meaning to the studied concepts. In general, the study developed in this article emphasizes the demonstration of results based on propositions adapted a priori, whose ability to be developed is aimed at establishing an "if...then" type of reasoning, making conjectures involving various knowledge already acquired and confirming such truths from a logical system, using definitions and propositions. Therefore, the demonstrations made in the scope of Synthetic Geometry will help to establish a connection with the equations obtained from the perspective of Analytical Geometry, serving as a consultation for students and professors of Analytical Geometry, thus avoiding sudden transitions between contents of degrees of distinct difficulties.
\end{abstract}

Keywords: Pure Geometry, Algebraic Thought, Conic Curves, Study of the Ellipse

\section{Introduction}

Mathematics from the dawn of human civilizations to the present plays an important role in society in general and particularly in the world of science and work.

As a science, Mathematics is in vitality having contributed to society since ancient civilizations, it is still present today in the highest spheres of scientific thought, as well as in various technological applications [1]. As an area of knowledge, it emerged in an intuitive way, through the satisfaction of the practical needs of man, as well as the analysis of the problems of objective reality. Its emergence occurred fundamentally with the awakening of geometry, with the need for man to measure land for agriculture.

With the emergence of geometric thinking, the ancient Egyptians, Chinese, Babylonians, Romans and Greeks began to use geometry for typography, navigation, astronomy and other practical applications. The Greeks sought to systematize the geometrical notions then known by establishing logical reasons for each notion and their interrelationship. Several mathematicians, such as Thales (600 BC), Pythagoras (540 $\mathrm{BC}$ ) and Aristotle (350 BC) developed a work of systematizing geometric notions and principles, which culminated in the book Euclid's Elements [2].

With this discovery by Euclid, the problems began to be solved from the point of view of Synthetic Geometry, which is characterized as the branch of Mathematics that is in charge of studying and synthetically constructing geometric shapes and places; it is the study of geometry without the use of coordinates.

According to Klein, F., in his book Elementary Mathematics from an Advanced Stendpoint, he states that 
synthetic or pure geometry is one that can be constructed axiomatically, with a logical-deductive treatment; that is, from a set of axioms or postulates (adapted a priori). Geometric constructions are based on demonstrations of logical propositions that are supported as a kind of links in a "chain of reasoning [3]".

According to Pickover, "in 1637, the French philosopher, René Descartes, published La géometrie, which shows how geometric shapes and figures can be analyzed through Algebra [4]." In this author's perspective, Descartes' work influenced the evolution of Analytical Geometry, a branch of mathematics that contemplates the representation of positions in a coordinate system in which mathematicians analyze these positions algebraically.

The perspective developed in this article is based on the vision of René Descartes, when he considered that "the algebraic steps in a proof should really correspond to a geometric representation [4]". According to Carl Boyer quoted by Pickover, "Descartes wanted to free geometry from the use of diagrams through algebraic procedures and give meaning to algebra operations through geometric interpretations. [4]" In this perspective, following the line of thought of René Descartes, this article aims to study the ellipse from the perspective of pure or synthetic geometry to the representation of points on a plane through the use of real numbers, as well as the representation and the classification of this conic curve through the use of equations.

In view of the above, the approach to geometric and algebraic thinking in mathematics is presented below, followed by a brief historical introduction on conic curves. Next, the construction of the ellipse using Nadandelin spheres is presented, followed by the analytical study of the ellipse, ending with the final considerations.

\section{Approach to Geometric and Algebraic Thinking in Mathematics}

Geometric and algebraic thinking are two forms of mathematical reasoning that are of capital importance for the study of Mathematics. For a fruitful study of mathematical approaches, it is necessary to look at these two ways of thinking and how they are characterized and developed. [5], in his master's thesis on "Analytical Geometry in the Plane", presented and defended at the University of Beira Interior, in Portugal, he assumes five levels on the geometric thought of Dutch researchers, Pierre Van Hiele and Dima Van Hiele-Geldof, whose fundamental focus is facilitating the ways of understanding spatial ideas through the hierarchy reflected in these levels, which are reproduced below.

1) Level 0: Visualization (Shapes - Shape Classes)

2) At this level, figures are recognized and named, based on their global and visual characteristics. At this level it is the appearance of the form that defines it.

3) Level 1: (Shape Classes - Shape Properties)
4) At this level, you start to get a sense of the properties that define each shape, the subject is able to think, for example, about what defines a rectangle (four sides, parallel opposite sides, opposite sides of the same length, four right angles, congruent diagonals).

5) Level 2: informal deduction (Properties of Forms Relationships between properties)

6) At this level, there is a greater ability to establish "If...then" reasoning and shapes can be classified using only a minimal amount of features. For example, "four congruent sides and at least one right angle" may be enough to define a square.

7) Level 3: deduction (Relationship between properties Deductive systems of properties)

8) At level 2, conjectures are produced involving the relationships between properties. Are these assumptions correct? They are true? When this analysis begins to occur, the subject begins to feel the need for a Logical System - with axioms, definitions, theorems, corollaries - from which other geometric truths can be deduced. The subject can clearly observe that the diagonals of a rectangle bisect each other, proceeding to demonstrate this observation through a series of deductive arguments.

9) Level 4 - rigor (Deductive property systems - Analysis of deductive systems)

At the highest level of Van Hiele Theory, the objects of attention are the axiomatic systems themselves, not just the deductions within a System. There is an appreciation of the distinctions and relationships between different axiomatic systems, for example, Spherical Geometry and Plane Geometry each have, its own set of axioms and theorems. From this level, result comparisons and confrontations between the different axiomatic systems of Geometry.

Associated with geometric thinking is algebraic which involves forming generalizations from experiences with numbers and operations, formalizing these ideas using a System of Meaningful Symbols and exploring concepts of pattern and function. Far from being a topic of little use in the real world, algebraic thinking covers all of mathematics and is essential to making it useful in everyday life.

According to J. Kaput (1942-2005) quoted Guimarães, "it is difficult to find an area of Mathematics that does not mainly involve generalizing and formalizing in some way. In fact, that kind of reasoning is at the heart of Pattern and Order Mathematics [5]."

It was from this perspective that René Descartes, joining these two ways of thinking mathematics in the 17th century, created Analytical Geometry, whose study of conic curves, where the ellipse is studied, is under his care [6].

\section{Brief Historical Introduction to Conic Curves}

Euclid's Elements (300 BC) dealt with plane figures that 
could be constructed with segments and circles, corresponding solid figures that could be constructed with portions of planes, such as prisms and regular polyhedrons, and the sphere [10]. But the classical Greeks also studied another class of curves which they called conic sections because they were originally obtained by cutting a cone with a plane. The resulting curves, the parable, the ellipse, and the hyperbola, were studied by Euclid but also later by another Greek geometer, Appollonius, who wrote an exhaustive treatise on these curves. "His main contribution was the fact that he managed to generate all the conics of a single twoleaf cone, simply by varying the inclination of the intersection plane [8]."

According to Santos Guimarães, quoted by Monteiro in his Master's dissertation, states that "in 1822 a Belgian mathematician named Germinal Pierre Dandelin (1794-1847) introduced a new idea that would help demonstrate the properties of conic sections. Adolphe Quelet, also Belgian, and Dandelin's colleague, was an important contributor to this work [9]." Dandelin's work consisted in showing that given a plane that intersects a cone, there are one or two spheres that are tangent to the plane and to the cone. This is why they are called Dandelin spheres. Working with the property of tangents to a circle, which says that "given a point outside a circle, it is possible to draw two lines that tangent it to different points, and whose distances to the given point are equal, Dandelin is able to find the foci of the ellipse and of hyperbole and simultaneously verify the respective properties" [9].
On the other hand, "Dandelin could not show the focal property for the parable, but Pierce Morton, in 1829, used a construction similar to Dandelin's to prove this property. Unlike the ellipse and the hyperbola, in the proof of the parable there will only be a tangent sphere to the cone and to the plane" [11].

\section{Construction of the Ellipse Using Ndandelin Spheres}

According to Monteiro "a study of the construction of the ellipse using Dandelin spheres follows, based on the reasoning presented by [9]."

Definition 2.1: (synthetic perspective of the ellipse)

The ellipse is the curve that results from the intersection of a straight circular cone with an oblique plane at its base, not parallel to any of the cone's generatrices.

Proposition 2.1:

Consider a straight circular cone and a plane $\pi$ that intersects it obliquely not parallel to the axis nor to any generatrix of the cone. We assume that there are two spheres $S_{1}$ and $S_{2}$ that simultaneously tangent the plane and the cone, see Figure 1. Let $F_{1}$ and $F_{1}$, respectively, be the points of intersection of the spheres $S_{1}$ and $S_{2}$ with the plane $\pi$, then any point $P \in \pi$ at the intersection with the surface of the cone is such that:

$$
d\left(P, F_{1}\right)+d\left(P, F_{2}\right)=d(R, Q)
$$

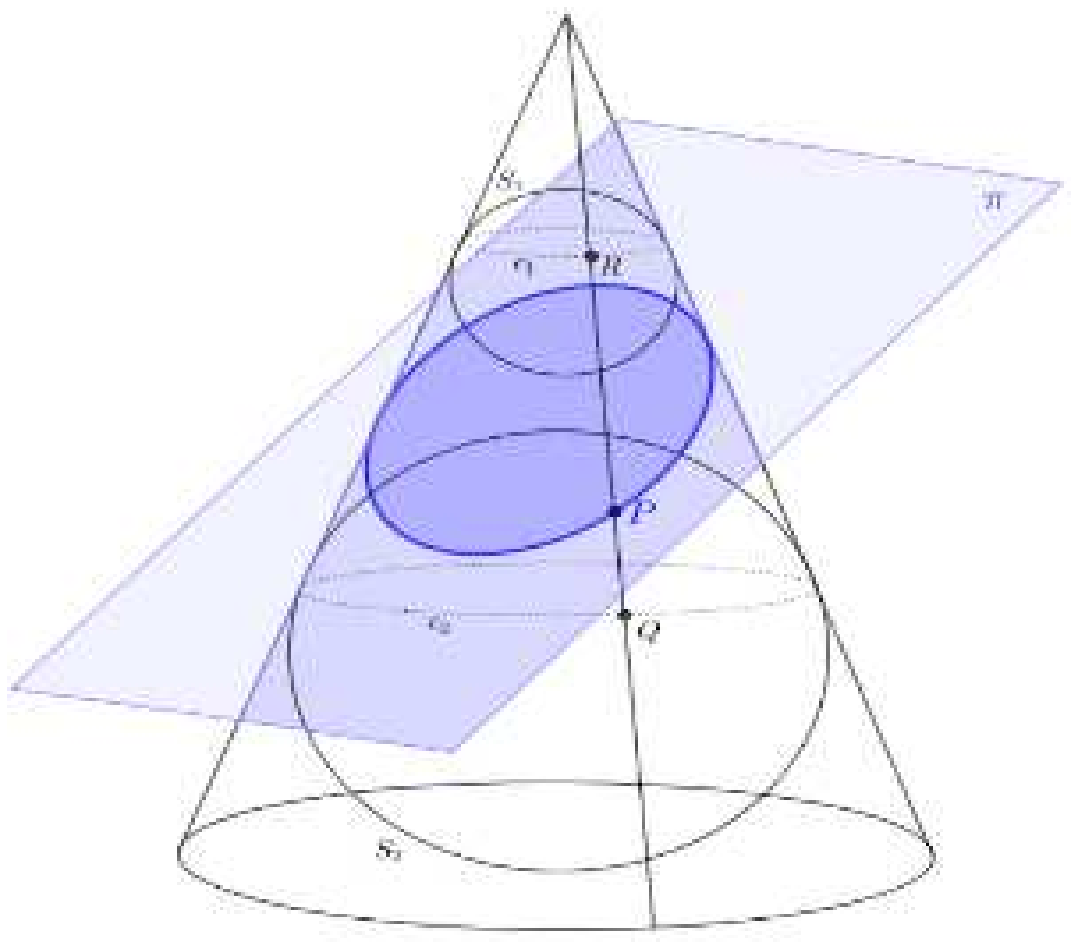

Figure 1. The ellipse as an intersection curve between the plane and the cone (a).

Proof:

Consider a straight circular cone and a plane $\pi$ that intersects it obliquely not parallel to the axis nor to any generatrix of the cone. 


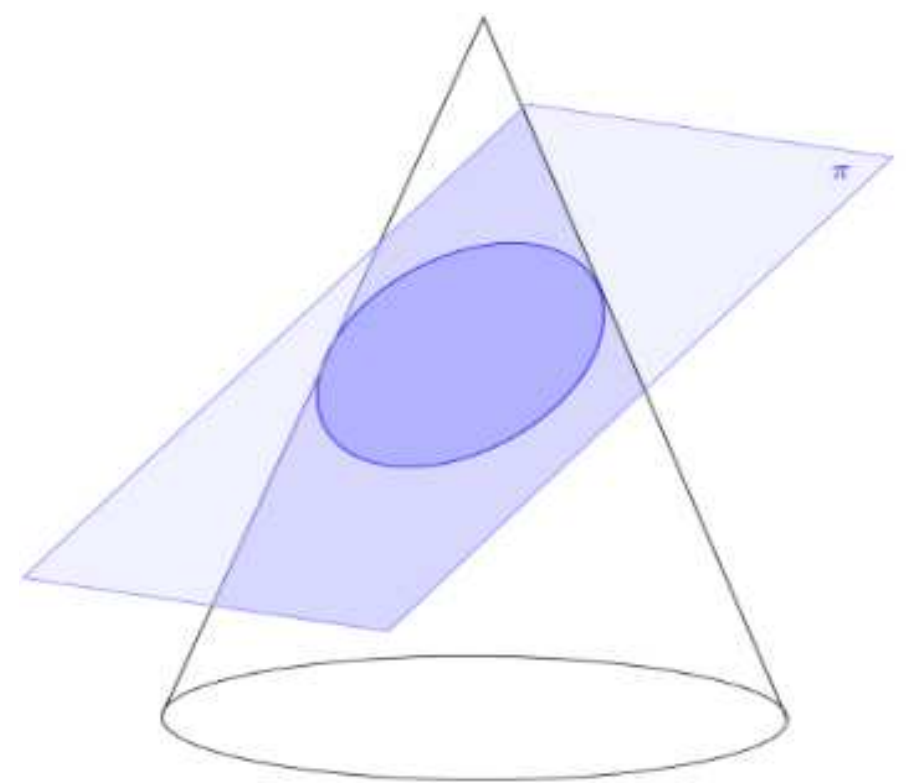

Figure 2. The ellipse as an intersection curve between the plane and the cone (b).

Let the circles $c_{1}$ and $c_{2}$, be tangent to all generatrices of the cone, the intersection of the cone surface with the spheres $S_{1}$ and $S_{2}$ respectively. Let $P \in \pi$ be a point at the intersection of the plane and the cone.

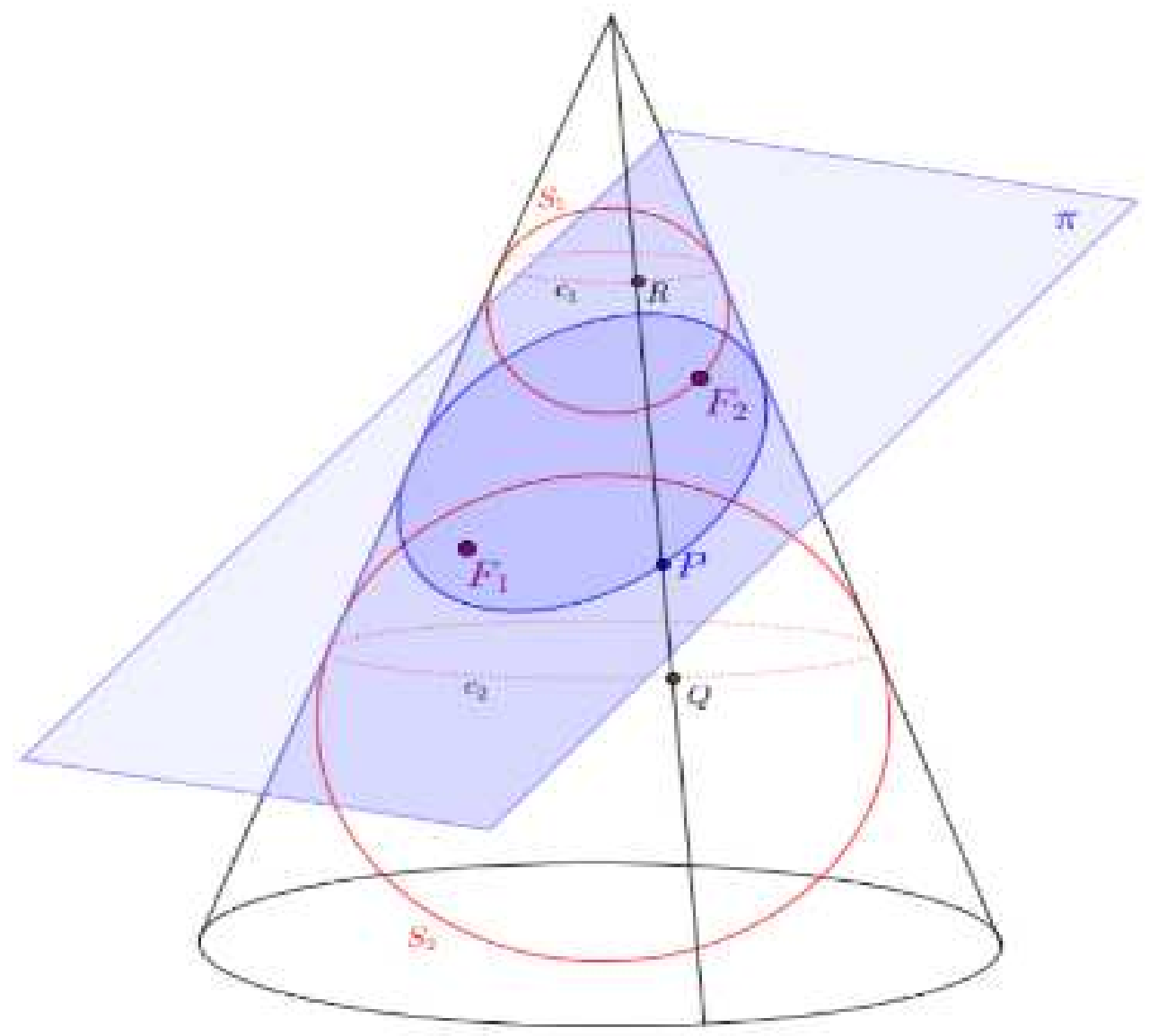

Figure 3. The ellipse as an intersection curve between the plane and the cone (c).

Let $R$ and $Q$ be the intersections of the generatrix of the cone that passes through $P$ with the circles $c_{1}$ and $c_{2}$ respectively. Let $\overline{R Q}$ be the length of the segment between $c_{1}$ and $c_{2}$ that passes through $P$, see Figure 3. Points $P, F_{1}$ and
$Q$ define a plane that intersects the Dandelin sphere $S_{2}$ on one circle and points $P, F_{2}$ and $R$ define a plane that intersects the Dandelin sphere $S_{1}$ on another circle. By the property of tangents to circles by an exterior point, we have: 

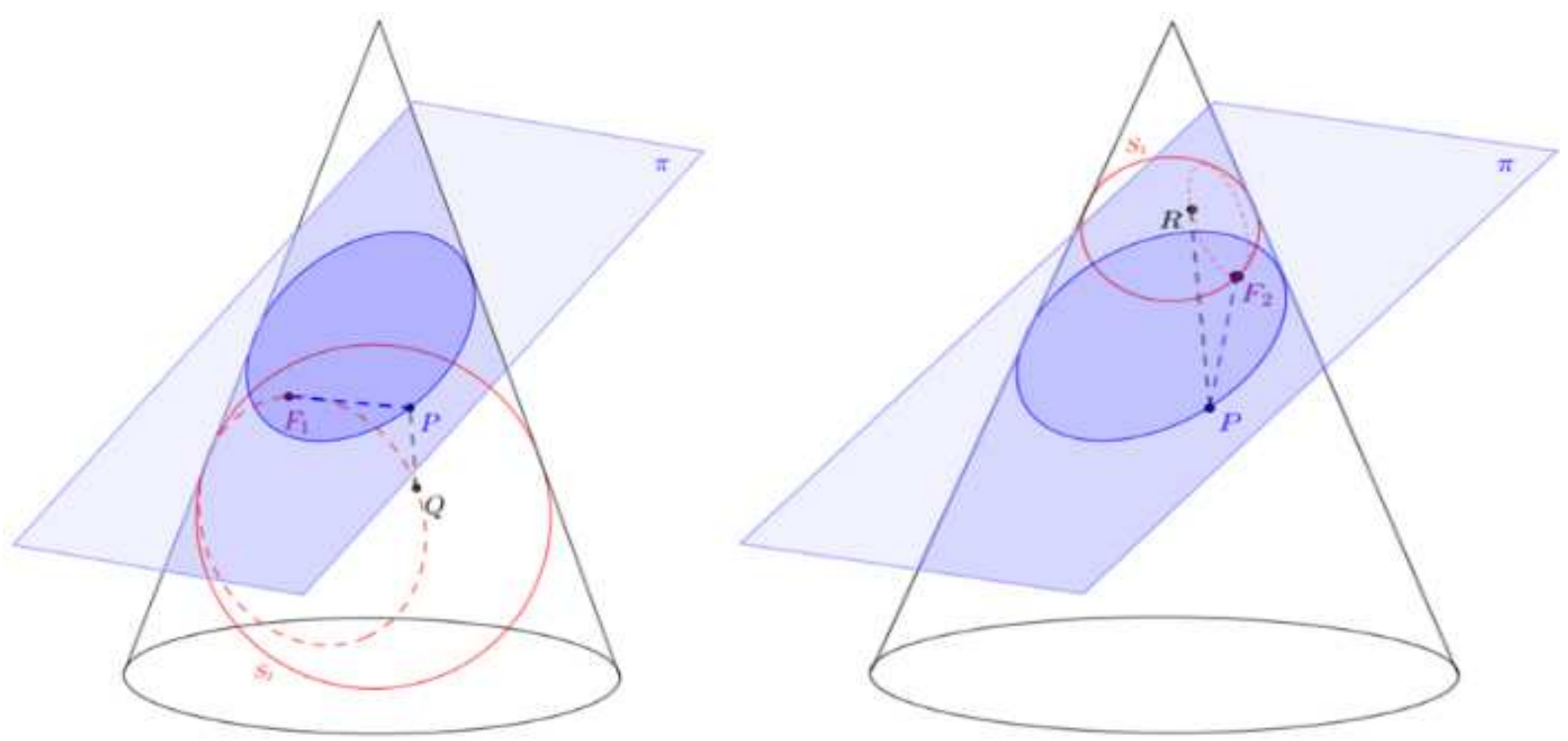

Figure 4. Property of tangents to circles.

$$
d\left(P, F_{1}\right)=d(P, Q) \text { e } d\left(P, F_{2}\right)=d(P, R) .
$$

Then:

$$
d\left(P, F_{1}\right)+d\left(P, F_{2}\right)=d(P, Q)+d(P, R)=d(Q, R)
$$

This sum will always be constant no matter where the point $P$ on the intersection of the plane with the cone is chosen. The result obtained represents the main property of the ellipse that makes it be considered a locus.

According to the thought of René Descartes, the result found can be studied in the representation of positions in a coordinate system, analyzing these positions algebraically. This approach is based on the area of knowledge called Analytical Geometry, as shown in the following section.

\section{Analytical Study of the Ellipse}

The following study was developed based on [9].

Definition 3.1: (analytical perspective of the ellipse)

An ellipse is the set of points on a plane whose sum of distances to two fixed points on the plane (focuses) is constant.

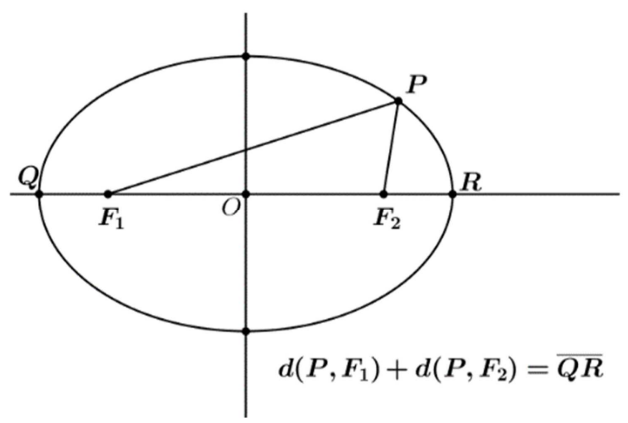

Figure 5. The ellipse in the plane with the foci on the xx axes.

Proposition 3.1:

Consider an $X$ and $Y$ rectangular coordinate system, in which an ellipse centered on the origin is represented. Let $P(x, y)$ be an arbitrary point belonging to this ellipse. Let $F_{1}(-c, 0)$ and $F_{2}(c, 0)$ be fixed points of the ellipse positioned on the abscissa axis. Consider $A_{1}(-a, 0)$ and $A_{2}(a, 0)$, vertices positioned on the axis of the ellipse containing the points $F_{1}$ and $F_{2}$, whose length is $2 a \cdot d\left(P, F_{1}\right)+d\left(P, F_{2}\right)=2 a$ then the locus that satisfies the set of points $P(x, y)$ is

$$
\frac{x^{2}}{a^{2}}+\frac{y^{2}}{b^{2}}=1
$$

Proof:

If $F_{2}$ has coordinates $\left.c, 0\right), c>0$, then $F_{1}$ has coordinates $(-c, 0)$. The distances from $P$ to $F_{1}$ and $F_{2}$ is $2 c$. Let's denote by $2 a$ the sum of the distances from $P$ to $F_{1}$ and $F_{2}$. By the definition of the ellipse $P(x, y)$ will belong to the Ellipse if:

$$
d\left(P, F_{1}\right)+d\left(P, F_{2}\right)=2 a
$$

By the formula of the distance between two points we have:

$$
\begin{gathered}
\sqrt{(x-c)^{2}+y^{2}}+\sqrt{(x+c)^{2}+y^{2}}=2 a \\
\sqrt{(x+c)^{2}+y^{2}}=2 a-\sqrt{(x-c)^{2}+y^{2}} \\
\left(\sqrt{(x+c)^{2}+y^{2}}\right)^{2}=\left(2 a-\sqrt{(x-c)^{2}+y^{2}}\right)^{2} \\
x^{2}+2 x c+c^{2}+y^{2} \\
=4 a^{2}-4 a \sqrt{(x-c)^{2}+y^{2}}+x^{2}-2 x c \\
+c^{2}+y^{2} \\
4 x c-4 a^{2}=-4 a \sqrt{(x-c)^{2}+y^{2}} \\
\left(x c-a^{2}\right)^{2}=a^{2}\left(x^{2}-2 x c+c^{2}+y^{2}\right) \\
x^{2} c^{2}-2 a^{2} x c+a^{4}=a^{2} x^{2}-2 a^{2} x c+a^{2} c^{2}+a^{2} y^{2} \\
x^{2} c^{2}-a^{2} x^{2}-a^{2} y^{2}=-a^{4}+a^{2} c^{2} \\
-x^{2}\left(a^{2}-c^{2}\right)-a^{2} y^{2}=-a^{2}\left(a^{2}-c^{2}\right)
\end{gathered}
$$


Knowing that $a>c$, and taking into account the Pythagorean theorem, we have: $b^{2}=a^{2}-c^{2}$. Then the equation (1), is reduced in shape: $b^{2} x^{2}+a^{2} y^{2}=a^{2} b^{2}$, dividing both members by $a^{2} b^{2}$, we have:

$$
\frac{x^{2}}{a^{2}}+\frac{y^{2}}{b^{2}}=1
$$

Note that the graph of the equation (2) is symmetric about both coordinate axes. When $y=0$,

$x= \pm a$, and when $x=0$, comes $y= \pm b$. So the ellipse intersects the axis of $x x$ in the stitches $A_{1}(-a, 0)$ e $A_{2}(a, 0)$ and intersects the axis of $y y$ in the stitches $B_{1}(0,-b)$ and $B_{2}(0, b)$. Thus, the segment $\overline{A_{1} A_{2}}$ is called the major axis of the ellipse and the segment $\overline{B_{1} B_{2}}$ is called the minor axis of the ellipse [12].

If we consider the focuses of the ellipse on the ordinate axis, at the points $(0,-c)$ e $(0, c)$ the equation stays:

$$
\frac{y^{2}}{a^{2}}+\frac{a^{2}}{b^{2}}=1
$$

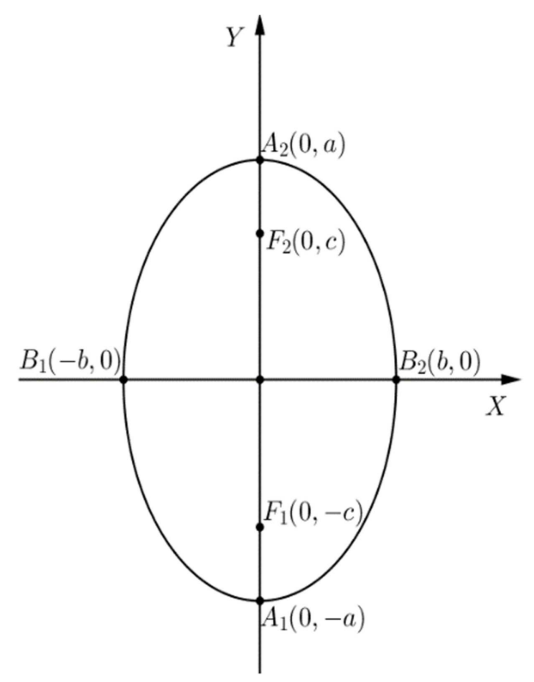

Figure 6. The ellipse in the plane with the foci on the y-axis [13].

The points on the ellipse do not move away from the foci indefinitely. We can use the equation of the ellipse (2) to deduce that its extension is limited, to do so, just solve the equation in order of $x$ or $y$ :

$$
\begin{aligned}
& x= \pm \frac{a}{b} \sqrt{b^{2}-y^{2}} \\
& y= \pm \frac{b}{b} \sqrt{a^{2}-x^{2}}
\end{aligned}
$$

Equations (4) and (5) show that $y^{2}$ cannot exceed $b^{2}$ and that $x^{2}$ cannot exceed $a^{2}$. That is, the admissible values are:

$$
\begin{aligned}
& -b \leq y \leq b \\
& -a \leq x \leq a
\end{aligned}
$$

In the case of equations (2) and (3) the vertices are respectively.

$A_{1}(-a, 0)$ and $A_{2}(a, 0)$, or $A_{1}(0,-a)$ and $A_{2}(0, a)$; $B_{1}(0,-b)$ and $B_{2}(0, b)$ or $B_{1}(-b, 0)$ or $B_{2}(b, 0)$, but the relationships between $a, b$ and $c$ do not change. The general equations of the ellipse when the axes are parallel to the coordinate axes and originate from a coordinate point $(h, k)$ are:

$$
\begin{aligned}
& \frac{(x-h)^{2}}{a^{2}}+\frac{(y-k)^{2}}{b^{2}}=1 \\
& \frac{(y-h)^{2}}{a^{2}}+\frac{(x-k)^{2}}{b^{2}}=1
\end{aligned}
$$

Observing equations (6) and (7), it is possible to see that both are grade 2 in both variables. You can develop the squares and be written in the following ways:

$$
\begin{aligned}
& A x^{2}+B y^{2}+D x+E y+F=0 \\
& A y^{2}+B x^{2}+D x+E y+F=0
\end{aligned}
$$

For an ellipse of focuses $(0, \pm c)$ ou $( \pm c, 0)$ and long axis $2 a$, the quantity is called the eccentricity of the ellipse.

$$
e=\frac{c}{a}
$$

Like $a>c$, the eccentricity is a number between 0 and 1 : $0<e<1$.

The shape of an ellipse depends on the value of its eccentricity. The longer the focal length of an ellipse, with fixed, the more the eccentricity approaches 1 and the shorter the focal length, the more the eccentricity approaches 0 [14].

By varying the eccentricity between 0 and 1, we have:

1. If $e=0$, This means that $c=0$, soon $b^{2}=a^{2}$ and therefore the two focuses coincide with the centre, in which case the ellipse is a circumference. If $e=1$ than $c=a$, soon $b^{2}=0$ and therefore, in this case we cannot define the ellipse by any of equations (2) and (3). This is the case where the graph is simply a straight line connecting the focuses [15].

\section{Conclusion}

The ideas presented in this article are based on the perspective of René Descartes, aimed at freeing geometry from the use of diagrams through algebraic procedures and giving meaning to algebraic operations through geometric interpretations. The deductions made from the point of view of synthetic geometry will help to understand the main property of the ellipse through which it is considered to be a locus.

This study will serve as a basis for understanding the main property that justifies the fact that the ellipse is considered a geometric locus in the context of Analytical Geometry.

\section{References}

[1] Cassela, EAD. (2020). Da Matemática nativa presente no pensamento das autoridades tradicionais da tribu Umbundu com respeito a forma do ondjango no Cuito/Bié-Angola para problemas isoperimétricos da Geometria Plana. European Reviw of Artistic Studies, v. 11, n. 2, p. 69-80. 
[2] Rich, B. (1972). Geometria Plana. Coleção Schaum. Brasil: Eitora Mc Graw-Hill, Ltda, p. 1.

[3] Klein, F. (1948). Elementary Mathematics from an Advanced Standpoint. Geometry, New York. Dover.

[4] Pickover, CA. (2009). O livro da Matemática. New York: NY 10016, VS, p. 136.

[5] Guimarães, RMS. (2014). Geometria Analítica no plano. Pensar e ensinar Geometria. Master's Thesis, Universidade da Beira Interior, Covilhã, Portugal, p. 3.

[6] Cassela, EAD., \& DE Nascimento, RM., (2020). Estudo da Circunferência à luz dos princípios axiomáticos de René Descartes. Um olhar ao context de Ensino-Aprendizagem da Escola Superior Pedagógica do Bié. Revemat, v. 15, p. 1-21.

[7] Boyer, CB (2003). História da Matemática. Edgard Blucher, São Paulo.

[8] Guimarães, CS. (2008). Matemática em nível Ime Ita. São José dos Campos. Vestseller, p. 11.

[9] Monteiro, RM. (2014). Resgate do teorema de Dandelin no estudo de cónicas com o Geogebra. Master's Thesis,
Universidade Federal do Espítrito Santo, Brasil. Disponível em: http://repositório.ues.br/handle/10/4819.

[10] Fuller. G., Connors, J., \& Levy, J. (2020). COVID-19 and its implications for thrombosis and anticoagulation. Blood, 135, $2033-2040$.

[11] Cassela. EAD (2018). Estudo da Geometria Analítica no Contexto cultural do Cuito. Master's Thesis, Universidade da Beira Interior Portugal.

[12] Cassela. EAD (2019). Abordagem sintética e analítica das cónicas. Uma perspectiva de Ensino em contexto. Editora Templários, Lisboa, Portugal.

[13] Santos, EC. \& Cassela, EAD. (2021). Interface entre a elipse e a circunferência: Contributo da etnomodelagem no ensino da Geometria Analítica por meio de cestaria. Matemática \& Ciência, v. 4, n. 1, p. 73-86.

[14] Alcopyan, A. V., Zaslavsky A. A (2007). Geometry of conics. American Matematical Society.

[15] Queiró, F., J., A elipse, a parábola e hipérbole - propriedades e aplicações (2010). Master's Thesis, Universidade Federal de Santa Catarina, Brasil. 